\title{
Planilandia: una aventura literaria en el mundo de la matemática
}

\author{
Flatland: a Literary Adventure inside \\ Mathematics World
}

Luis Ricardo Martínez*

Los nombres no designan a las cosas: las envuelven, las sofocan. Pero las cosas rompen sus envolturas de palabras y vuelven a estar ahí, desnudas, esperando algo más que los nombres.

Roberto Juarroz

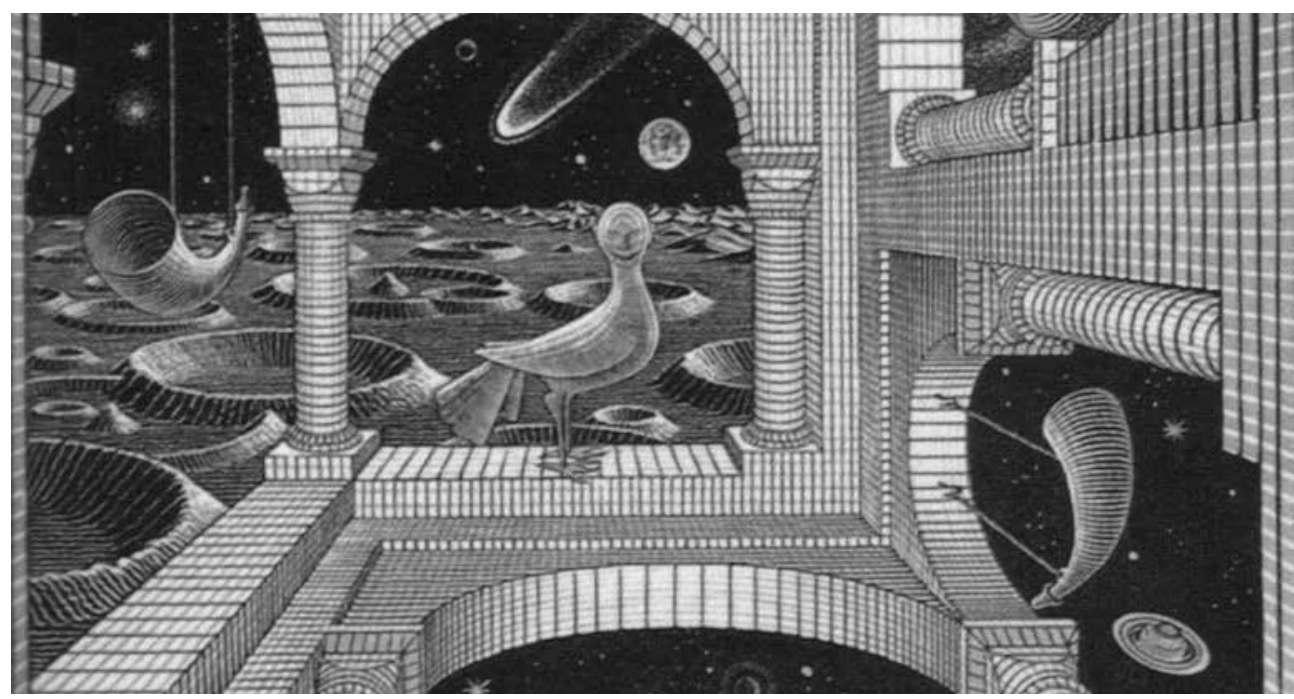

\section{Resumen}

Se presenta una reseña breve y analítica, con algunos comentarios en el ámbito político, de esta novela clásica, Planilandia, un texto del siglo XIX (1884), escrita por Edwin Abbott.

Palabras clave: matemáticas, dimensión, novela.

Citar este artículo como: Martínez, L. (2016). Planilandia: una aventura literaria en el mundo de la matemática. Revista Papeles, 8(16), 35-40.

Fecha de recibido: septiembre de 2016.

Fecha de aceptación: diciembre 15 de 2016.

* Economista de la Universidad Nacional de Colombia. Miembro del laboratorio de investigación LE2P -Laboratorio espacio, economía y poder, UNAL. Un hombre enamorado de la sabiduría oriental, ya sea Gandhi o el budismo. Correo electrónico: lurmartinezra@unal.edu.co 


\begin{abstract}
A brief and commented review, with some commentaries about political issues, on a classic novel, Flatland. This text was written by Edwin Abbott in 1884.
\end{abstract}

Keywords: mathematics, dimension, novel.

Planilandia ${ }^{1}$, obra de aventura ${ }^{2}$ y ficción matemática del escritor inglés Edwin A. Abbott, recrea la sociedad inglesa de la era victoriana transportando al lector a un mundo geométrico, en el que el plano euclidiano comprende la totalidad del universo de su protagonista. Un respetado cuadrado de Planilandia se verá atrapado en la frustrante paradoja de poder conocer universos más allá de sus dos dimensiones para fallar en todo intento por compartir ese conocimiento con sus conciudadanos.

Planilandia es el espacio en el que comienza la aventura, un mundo de dos dimensiones en el que todos sus habitantes son figuras geométricas superficiales, regidas por estrictas leyes naturales y sociales. Todo lo que ven en el mundo tiene siempre la apariencia de una línea, una línea de grosor infinitesimal y las únicas direcciones en las que se mueven son los cuatro puntos cardinales. La lluvia cae siempre de norte a sur por lo que sus casas están orientadas con el techo siempre hacia el norte.

Coged, por ejemplo, un triángulo equilátero, que representa entre nosotros un comerciante de la clase respetable. La fig. 1 representa al comerciante tal como le veríais cuando os inclinaseis sobre él y le miraseis desde arriba; las figs. 2 y 3 representan al comerciante como le veríais al acercaros al nivel de la mesa y ya

1 Título completo del texto reseñado: Planilandia, una novela de muchas dimensiones. Publicado en 1999. Título original en inglés: Flatland, $A$ romance of many dimensions de Edwin Abbott. Publicado originalmente en 1884.

2 La palabra novela, traducida del original romance en inglés, hace referencia más exactamente a una historia de aventura, y no tanto a una novela en el sentido estricto del género literario. casi en él; y si vuestros ojos estuviesen al nivel de la mesa (y así es como le vemos nosotros en Planilandia) no veríais nada más que una línea recta (p. 11).

Figura 1. Triángulo visto como una línea

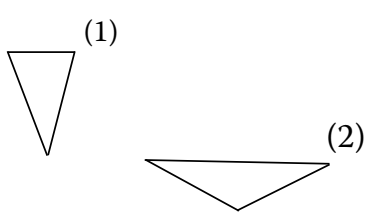

Fuente: ilustraciones de Abbott en la versión original

Los habitantes de Planilandia están claramente estratificados según su número de lados y sus ángulos, todas las figuras son convexas y conformadas por líneas rectas. La clase más baja es la de los triángulos isósceles, soldados y obreros puntiagudos con dos lados iguales; mientras su base sea más pequeña, más baja será su posición en la sociedad. Le siguen en orden ascendente los triángulos equiláteros, la clase media, similar a la clase media común victoriana; los cuadrados y pentágonos son los profesionales y caballeros, la middle class victoriana; a esta le siguen la nobleza conformada por hexágonos, y por figuras de más de seis lados. Por último, una persona que tiene tantos lados, y sus ángulos son tan amplios y numerosos, que no se distingue de una circunferencia, hace parte del grupo sacerdotal, la clase más alta en toda la sociedad en Planilandia. En contraste, el grupo que ocupa el puesto más bajo en el texto de Abbott es la mujer. Conformadas por una solo línea recta, como un triángulo isósceles tan degradado que su base tiende a cero, la mujer es el más insignificante de las personas que habitan Planilandia. 
Todas estas figuras están compuestas por lados iguales, menos los isósceles, o por lo menos eso aparentan. El resto de figuras, "deformes", compuestas por lados desiguales, son excluidas de la sociedad o ejecutadas, y no tiene cabida en esta.

La idea de contar una historia usando figuras geométricas como personajes y retratar el mundo por medio de analogías matemáticas parece improbable en un principio. Sin embargo, en la Inglaterra de Abbott la relación entre la realidad y la geometría se hace más estrecha que nunca antes en la historia de la humanidad. La era victoriana es la época del nacimiento de la ingeniería de precisión. Las fábricas inglesas son ahora capaces de producir en masa objetos metálicos medidos y cortados con absoluto detalle. La realidad reproduce la geometría. Abbott, en sentido inverso, plasma esa realidad de regreso en el papel. La caricatura geométrica de una época rígida y contradictoria permite ver, sin causar sobresaltos, la realidad moldeada por grandes desigualdades. La mujer como ser despreciable, el obrero relegado al lugar más bajo de la sociedad, la nobleza y la naciente burguesía, disfrutando del orden rígido e invariable, en apariencia.

Sin embargo, estas observaciones trascienden al autor y a su época. Planilandia pregunta por la relación existente entre las jerarquías establecidas, sus beneficiarios y perjudicados, y la resistencia que de estas emana para abrazar el cambio. Estas relaciones son universales a toda sociedad humana. Lo establecido permite eficiencia, certidumbre y eficacia a costa de dejar de lado lo posible. Lo posible permite crecer, innovar y expandir los límites humanos a costa de altos sacrificios, vidas, riquezas y tiempo.

A medida que el número de lados decrece, desde los sacerdotes, hasta las mujeres, la inteligencia y capacidad disminuye en la medida en que la magnitud de sus ángulos disminuye. El conocimiento del mundo, en especial de las matemáticas y la geometría, así como la capacidad de identificar a otros ciudadanos identificando sus ángulos por métodos más complejos, se concentra en las clases superiores; mientras las clases más bajas, los isósceles y las mujeres, están desprovistas de conocimiento, y su vida es servil y miserable. Los círculos, polígonos, hexágonos, pentágonos y cuadrados mejor educados pueden distinguir el ángulo de otra persona, gracias a la opacidad o brillantez con que este se ve mirado de frente. Las clases más bajas requieren del tacto para hacer la identificación respectiva.

Suponed que veo que se acercan dos individuos cuyo rango deseo determinar. Supongamos que son un comerciante y un médico, o, dicho de otro modo, un triángulo equilátero y un pentágono: ¿cómo puedo distinguirlos?

Figura 2. Identificación visual de las figuras
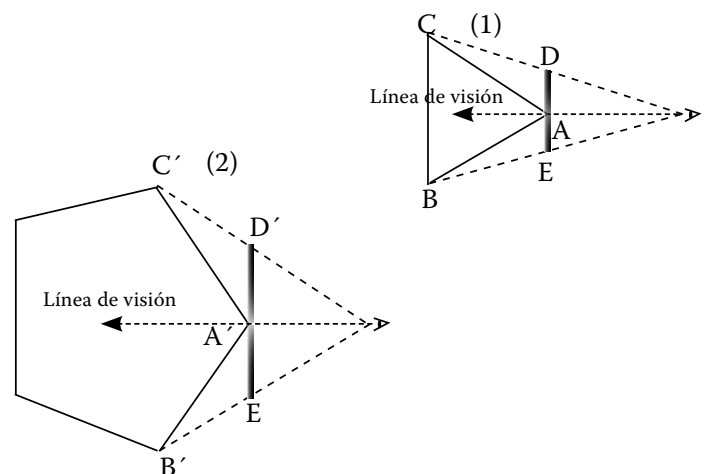

Fuente: ilustraciones de Abbott en la versión original

¿Qué veré ahora en el caso (1) del comerciante? Veré una línea recta DAE, en la que el punto medio (A) será muy brillante, porque es el que está más cerca de mí; pero a ambos lados la línea se hará enseguida borrosa, debido a que los lados $\mathrm{AC}$ y $\mathrm{AB}$ se pierden rápidamente en la niebla y lo que a mí me parecen las extremidades del comerciante, es decir D y E, serán realmente muy imprecisos.

Por otra parte, si pasamos (2) al médico, aunque también veré en este caso una línea (D'A'E') con un centro brillante (A'), se hará borrosa menos rápidamente, porque los lados 
$\left(A^{\prime} C^{\prime}, A^{\prime} B^{\prime}\right)$ se pierden menos rápidamente en la niebla: y lo que a mí me parecen las extremidades del médico, es decir, D' y E', no serán tan tenues como las extremidades del comerciante (pp. 22-23).

Sin embargo, las cosas no han sido siempre así. Planilandia posee una historia de rebelión e inconformismo, en la que el uso del color como forma de identificación estuvo cerca de acabar con el orden establecido. Los círculos, aprovechando su inteligencia, fraguaron la traición entre las clases bajas, retomaron el control y abolieron para siempre el uso del color en toda Planilandia. El orden jerárquico se entendió, era necesario para mantener la paz y garantizar el funcionamiento y la seguridad.

EN CUANTO A la doctrina de los círculos, puede resumirse brevemente en una sola máxima: «Atiende a tu configuración». Toda su doctrina, ya sea política, eclesiástica o moral, tiene por objeto la mejora de la configuración individual y colectiva... con especial referencia a la configuración de los círculos, a la que todos los demás objetivos se hallan subordinados (p. 35).

Dicho orden jerárquico se mantendrá, a pesar de que grandes segmentos de la sociedad estén descontentos o se sientan menospreciados. Para los círculos, la ilusión de ascenso en la sociedad por medio de la mejora progresiva en la descendencia es suficiente incentivo para mantenerse oprimido.

Abbott dedica casi la mitad de su libro a describir con detalle la configuración espacial y social de Planilandia; esto no es gratuito. Después de describir la rigurosidad de su mundo, natural y político, el viaje que en un sueño comienza, a través de diversos universos, hará más palpable lo improbable de sus visiones y experiencias, y se acentuará la dificultad que implicará la tarea de comunicar a sus propios congéneres de dos dimensiones lo que ha visto más allá de su universo, e incluso a sí mismo a medida que el recuerdo de sus experiencias se hace cada vez más lejano.
El conocimiento fabricado con lenguaje es a su vez contenido y continente. Le permite construir lo que va comprendiendo pero solo en la medida en que cabe en aquello que ya conoce. Paradójicamente, en la medida en que construye dentro de lo ya construido, el límite de dicho conocimiento, de dicho lenguaje, se expande.

Inadvertidamente, en un sueño, el cuadrado protagonista visita un mundo de una sola dimensión. Un mundo que para él es factible de comprender, dado que está compuesto por menos elementos de los que componen su mundo. Sin embargo, la forma de pensar y concebir el universo de los habitantes de Linealandia se le hace escurridiza. Más aún, sus intentos por explicarles a estos seres unidimensionales la naturaleza de su propia experiencia bidimensional son inútiles. $\mathrm{Ni}$ siquiera su materialización física en dicho mundo es suficiente para hacerle entender a un ser unidimensional la existencia de una realidad extendida, más allá de sus experiencias lineales. El espacio no cabe ni en la mente ni en el mundo de estos segmentos animados.

Figura 3. Cuadrado en el mundo lineal

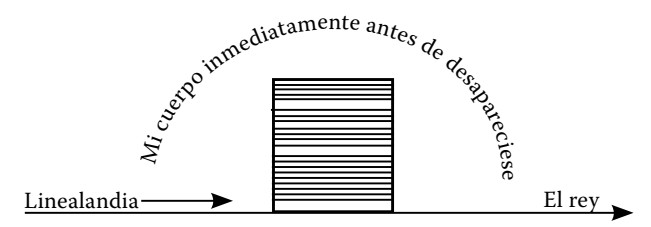

Fuente: ilustraciones de Abbott en la versión original

Una vez despierto, el cuadrado repetirá la experiencia de extrañeza que emana de intentar explicar, y comprender, aquello para lo que no se tiene ni palabras ni experiencias referentes. Será ahora él el que experimente la visita de un ser proveniente de una dimensión de mayor complejidad, las tres dimensiones de Espaciolandia, una esfera que al ingresar a su universo bidimensional solo podrá ser observado por el cuadrado, en un principio, como un círculo que varía su tamaño. 
Figura 4. Esfera en el mundo superficial

(1)

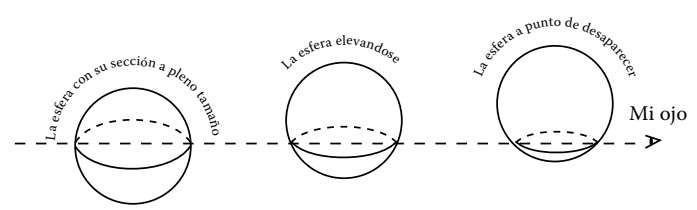

Fuente: ilustraciones de Abbott en la versión original

Será necesario que el cuadrado se eleve por encima de su realidad para poder comenzar a percibir, y construir en su experiencia, una realidad más compleja que la que ha podido vivir hasta ahora. No será suficiente con contemplar por primera vez con sus ojos la realidad del volumen, ni con escuchar de boca de la esfera, la realización teórica del desplazamiento del plano. El cuadrado no tendrá suficiente tiempo en Espaciolandia como para reconstruir en su mente una percepción propia de la profundidad. Al final del corto viaje de tres dimensiones, solo se quedarán en su mente las analogías y descripciones teóricas de lo que vio. Por el contrario, las cosas que más sentido tendrán para el cuadrado serán las referentes a su mundo plano. Al divisar su casa desde arriba por primera vez, el cuadrado verá con sus ojos algo que ya ha visto durante años en su mente. La imagen de lo conocido, aún visto desde otra dimensión, se hace evidente y digerible por su mente superficial.

Figura 5. Vista de una casa de Planilandia desde la perspectiva de Espaciolandia

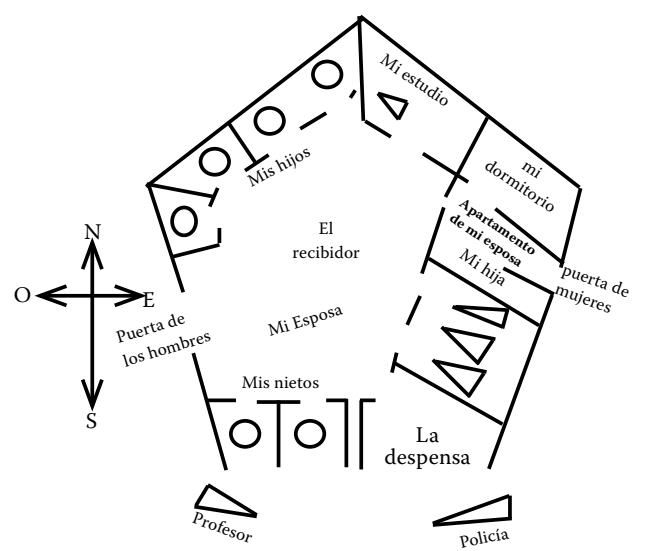

Fuente: ilustraciones de Abbott en la versión original
Como el cuadrado era diestro en geometría y matemáticas no tardó en conjeturar acerca de la existencia de otras dimensiones, más allá de las tres que se le habían revelado. Supuso que era plausible conjeturar acerca de la existencia de una cuarta y quinta dimensión, y en general, de la existencia de innumerables dimensiones. El acceso a unos pocos universos desconocidos le permitió pensar, por primera vez, en la existencia de otros universos posibles sin la necesidad de visitarlos. El conjunto de lo desconocido se había ampliado más allá de lo desconocido por conocer. La ausencia de la experiencia fue subsanada por el acceso a un universo aún más grande que cualquier otro, el universo de Pensamientolandia.

Figura 6. El pensamiento y el saber en Planilandia

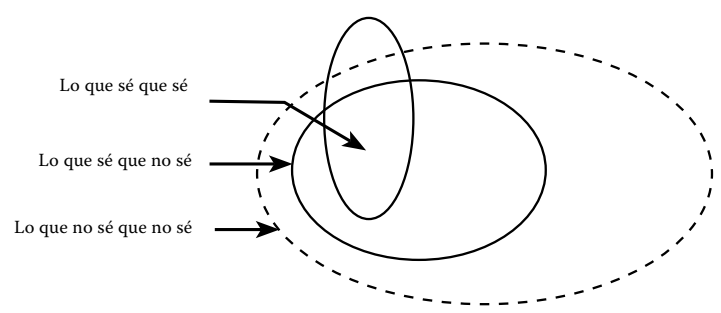

Fuente: ilustraciones de Abbott en la versión original

Es posible pensar que el conjunto de conocimientos que poseemos sea finito. De igual manera no será difícil convencernos de que el conjunto de lo que sabemos que ignoramos es a su vez finito. Sin embargo, el conjunto de aquello que ignoramos que no sabemos es más difícil de definir, en parte porque en la medida en que indagamos sobre el conjunto de lo ignorado, lo ignoramos un poco menos. A pesar de esto, es también factible pensar que el conjunto de lo que no sabemos que sabemos sea infinito o por lo menos suficientemente grande como para nuca abarcarlo por completo. Por último, vale la pena preguntarse si todo aquello que se encuentra actualmente en el conjunto de lo que sé, está contenido en el conjunto de lo que puedo saber, dado que, es probable que aquello que desconozco hoy, 
pero sabré mañana, excluya en el futuro parte de mi conocimiento actual, señalándolo como falso conocimiento.

De vuelta en Planilandia, el cuadrado se sentía abrumado por su experiencia espacial y sin saber qué hacer con este nuevo conocimiento. Luego tuvo una nueva visión en un sueño. La esfera aparecía nuevamente, pero esta vez lo llevaba a un mundo más estrecho y reducido que Linealandia, Puntilandia, un universo sin ninguna dimensión. El único habitante era un solitario punto. Era lo único que cabía en un universo infinitesimalmente pequeño, y esa sensación de ser todo lo que ocupaba el universo, de ser el universo mismo, le daba la sensación de grandeza y plenitud a este punto; no era un rey, era el dios mismo del universo. Por más que el cuadrado intentó abstraer al punto de su realidad, era imposible para él concebir la existencia misma de algo externo a sí mismo, a su universo.

El cuadrado comprende entonces que tiene que dar a conocer la doctrina de las tres dimensiones y que el limitado conocimiento del mundo bidimensional debe ser actualizado. Dedica el resto de sus energías a esta tarea y, sin embargo, como es de esperarse, la tarea se le hace imposible. El lenguaje no fue suficiente para explicar la tercera dimensión, cuando el cuadrado repetía "hacia arriba, no hacia el norte", la imagen que él tenía en su cabeza era totalmente distinta a la que sus interlocutores interpretaban.

Por último, no es únicamente la dificultad para explicar las tres dimensiones lo que hace que su intento por trasmitir este conocimiento fracase. La necesidad de mantener el orden jerárquico establecido por los círculos se ve amenazada por la posibilidad de la existencia de un orden superior, más allá de la sabiduría de los sacerdotes y que además cuestionaría la naturaleza del orden mismo. Lo que se daba sentado como cierto era susceptible de ser mirado desde otra perspectiva, esta otra perspectiva no es entonces solo física, sino que, al relativizar la forma, relativiza la interpretación que de esta se hace y pone en duda la rigidez de lo que se entiende en Planilandia por natural. El cuadrado es confinado a una celda en donde pasa los años y anhela poder elevarse a través de la tercera dimensión y pasar por encima de las paredes, de infinitesimal altura, que lo retienen. Desde su celda escribe las memorias que titula Planilandia.

En la aventura que resulta de una ficción geométrica, Abbott describe la realidad de su nación mientras pregunta, en un plano elevado, por nuestra capacidad por aprender en zonas lejanas a nuestro conocimiento. La relación entre realidad y matemática invita a pensar en la relevancia de la abstracción como manera de llegar a relacionarnos con la realidad, como manera de concebir lo inimaginado, como vital método de aventurarnos hacia lo desconocido. Indaga también por aquello que podemos no estar viendo, cegados por lo que vemos. Por cómo, encerrados en lo que sabemos, limitamos nuestras oportunidades de aprender, y sobre todo, de comprender al otro.

\section{Referencias}

Abbott, E. (1999). Planilandia, una novela de muchas dimensiones. Barcelona: Torre de viento. 